\title{
Composição corporal de idosas diabéticas tipo 2: antropometria vs absorcimetria de Raios-X de dupla energia
}

\author{
Body composition of elderly women with type \\ 2 diabetes: anthropometry vs dual-energy \\ $X$-ray absorptiometry
}

Waléria Christiane Rezende FETT ${ }^{1}$

Carlos Alexandre FETT ${ }^{1}$

Júlio Sergio MARCHINI²

Júlio Cesar MORIGUTI

Eduardo FERRIOLLI ${ }^{3}$

\section{R E S U M O}

\section{Objetivo}

Comparar a antropometria ao exame de Raios-x de dupla varredura para estimativa da composição corporal de idosas diabéticas tipo 2.

\section{Métodos}

A composição corporal de 51 voluntárias de 60 a 70 anos de idade e índice de massa corporal de 19 a $43 \mathrm{~kg} / \mathrm{m}^{2}$ foi avaliada pelas medidas antropométricas das dobras cutâneas do tríceps, suprailíaca e coxa e associadas ao exame de Raios-x de dupla varredura, considerado o padrão ouro. Os intervalos de concordância de Bland e Altman foram os valores do desvio-padrão do coeficiente de variação do exame de Raios-x de dupla varredura e os valores da antropometria deviam estar nessa faixa. Foram comparadas as médias (teste $t$ de Student) e feita a correlação de Pearson.

\section{Resultados}

Os intervalos de concordância foram os seguintes: peso $(\mathrm{kg})$ Média $(\mathrm{M})=0,3$, Desvio-Padrão $(\mathrm{DP})=0,1$, massa corporal magra $(\mathrm{kg}) \mathrm{M}=3,0, \mathrm{DP}=1,9$, massa corporal gorda $(\mathrm{kg}) \mathrm{M}=5,0, \mathrm{DP}=3,1$, percentual de gordura corporal ( $\% \mathrm{GC}) \mathrm{M}=5,0, \mathrm{DP}=3,1$. As médias entre os métodos eram iguais e estavam dentro do intervalo de concordância;

$\overline{1}$ Universidade Federal de Mato Grosso, Faculdade de Educação Física. Av. Fernando Correa da Costa, s/n., Ginásio de Esportes, Cidade Universitária, 78068-900, Cuiabá, MT, Brasil. Correspondência para/Correspondence to: W.C.R. FETT. E-mail: <wcrfett@hotmail.com>.

${ }^{2}$ Universidade de São Paulo, Divisão de Nutrição Clínica. Ribeirão Preto, SP, Brasil.

3 Universidade de São Paulo, Faculdade de Medicina de Ribeirão Preto, Divisão de Clínica Médica Geral e Geriatria, Departamento de Clínica Médica. Ribeirão Preto, SP, Brasil. 
696 | W.C.R. FETT et al.

em média, a antropometria superestimava a massa corporal magra em 2,31 kg, e subestimava a massa corporal gorda e o percentual de gordura corporal em -0,93 kg e -2,7\% respectivamente; a correlação entre as estimativas eram significativas ( $P, r=0,99 ; M C M, r=0,79 ; M C G, r=0,93 ; \% G C, r=0,72 ; p<0,0001)$.

\section{Conclusão}

Os resultados da antropometria e do exame de Raios-x de dupla varredura foram consistentes. Embora tivesse parte dos resultados da massa corporal gorda e do percentual de gordura corporal fora do intervalo de concordância, os outros resultados foram correspondentes, sugerindo o uso da antropometria para avaliação da composição corporal nessa população.

Termos de indexação: Composição corporal. Diabetes mellitus tipo-2. Idoso.

\section{A B S T R A C T}

\section{Objective}

This study aimed to compare anthropometry with dual-energy X-ray absorptiometry for estimating the body composition of elderly women with type 2 diabetes.

\section{Methods}

The body composition of 51 volunteers aged 60 to 70 years, with body mass indices ranging from 19 and $43 \mathrm{~kg} / \mathrm{m}^{2}$, was determined by anthropometry (triceps, suprailiac and thigh skinfold thicknesses) and dual-energy $X$-ray, which is considered the gold standard. The Bland-Altman limits of agreement were the standard deviations of the dual-energy $X$-ray coefficient of variation and the anthropometric values should be in this range. The means were compared by the Student's t-test followed by the Pearson's correlation.

\section{Results}

Limits of agreement: weight $(W, \mathrm{~kg}) M=0.3, S D=0.1$, lean body mass (lear body mass, $\mathrm{kg}$ ) $M=3.0, S D=1.9$; fat body mass (fat body mass, $\mathrm{kg}$ ) $M=5.0, S D=3.1$, percentage of body fat (\% body fat) $M=5.0, S D=3.1$. The means of the two methods were the same and within the limits of agreement. Anthropometry tended to overestimate lear body mass by $2.31 \mathrm{~kg}$ and underestimate fat body mass and \% BF by $-0.93 \mathrm{~kg}$ and $-2.7 \%$, respectively. The estimates correlated significantly $(W, r=0.99$; lear body mass, $r=0.79$; fat body mass, $r=0.93$; $\%$ body fat, $r=0.72 ; p<0.0001$ ).

\section{Conclusion}

The anthropometric and dual-energy $x$-ray results were consistent. Although some lear body mass and \% body fat results were beyond the limits of agreement, the others were within. Therefore, anthropometry can be used to assess the body composition of this population.

Indexing terms: Body composition. Diabetes mellitus, type 2. Aged.

\section{N T R O D U ÇÃ O}

O diabetes mellitus tipo 2 é caracterizado como uma desordem metabólica devida à deficiência insulínica e está associado a doença coronariana, hipertensão e dislipidemias ${ }^{1}$. Devido a essas complicações, o diabetes tipo 2 tem alto custo para os sistemas de saúde pública²; nos Estados Unidos, o custo estimado foi de U\$174 biIhões em $2007^{3}$. No Brasil houve aumento no número de hospitalizações por diabetes, proporções superiores às hospitalizações por todas as causas, o que pode traduzir o aumento da sua preva- lência ${ }^{4}$. O diabetes mellitus tipo 2 atinge em torno de 171 milhões de pessoas em todo o mundo, sendo estimado um aumento para 366 milhões de portadores em 2030. Entretanto, o aumento tem sido maior do que as previsões ${ }^{5}$.

O aumento da prevalência do diabetes tipo 2 no Brasil foi associado à obesidade e ao enveIhecimento populacional $^{6}$. Em um estudo multicêntrico brasileiro foi verificado aumento da prevalência do diabetes - similar ao da intolerância à glicose -, que foi associado a fatores ambientais e modificações do estilo de vida, como a adoção da dieta ocidental e do sedentarismo 7 . Além disso, 
o envelhecimento per se causa mudanças na composição corporal com aumento da Massa Corporal Gorda (MCG) e redução da Massa Corporal Magra $^{4}$ (MCM). Essas alterações estão associadas à redução da capacidade funcional e ao aumento de doenças em geral ${ }^{8}$.

Em torno de $80 \%$ a $90 \%$ dos diabéticos são do tipo 2 e, desses, mais de $80 \%$ são obesos ${ }^{9}$. Todavia, a relação precisa entre MCM e a MCG para determinação das limitações funcionais não estão definidas porque os dados são controversos $^{10}$ e os métodos não dão relações precisas entre si, sendo polêmico qual seria o melhor para determinada população ${ }^{11}$.

Um dos métodos mais precisos, não invasivo e rápido (5 a 10 minutos por exame) para avaliação da composição corporal é o exame de Raios-x de dupla varredura (DXA, dual-energy $X$-ray absorptiometry) ${ }^{11}$. É seguro e requer o mínimo de cooperação do avaliado, sendo o erro da medida do percentual de gordura próximo a $3 \%{ }^{12}$. A correlação do DXA para estimativa da MCM $\left(r^{2}=0,98\right)$ é alta quando comparada ao método de quatro compartimentos e também com a massa muscular estimada pela tomografia computadorizada $\left(r^{2}=0,86 \text { a } 0,96\right)^{13}$.

A disponibilidade desse método, todavia, se restringe aos hospitais e às clínicas especializadas, reduzindo sua aplicabilidade no nível populacional. Porém, a antropometria é de fácil aplicabilidade clínica devido ao fácil uso dos instrumentos, baixo custo e por fornecer informações sobre a composição corporal e os fatores de risco para doenças crônicas não-transmissíveis ${ }^{14}$, podendo auxiliar no tratamento e prevenção do diabetes ${ }^{2}$. Métodos mais precisos como a pesagem hidrostática, o próprio DXA, a ressonância magnética e o método da água deuterada são muito caros, o que torna a antropometria atraente.

Embora alguns métodos e/ou equações antropométricas para estimar a composição corporal de indivíduos idosos sejam validados, ainda é difícil escolher o melhor. Estudos que comparem a antropometria a métodos mais precisos são necessários, com o intuito de identificar suas limi- tações e aplicações ${ }^{8}$. Entretanto, ainda não existem estudos de validação para o uso da antropometria em idosas diabéticas. Assim, o objetivo deste estudo foi avaliar e comparar a composição corporal obtida pelo DXA e pela antropometria, utilizando-se uma equação de amplo espectro populacional em idosas diabéticas do tipo 2 .

\section{M É TOD OS}

O estudo foi realizado no Ambulatório de Diabetes do Hospital das Clínicas da Faculdade de Medicina de Ribeirão Preto da Universidade de São Paulo (HCFMRP-USP). Cinquenta e uma voluntárias com idade entre 60 e 70 anos, Índice de Massa Corporal (IMC) variando de 19 a $43 \mathrm{~kg} / \mathrm{m}^{2}$ e portadoras de diabetes tipo 2 foram selecionadas pelo banco de dados do HCFMRP-USP. Depois dessa seleção, as voluntárias foram contatadas por telefone ou pessoalmente no Ambulatório de Diabetes, sendo então explicados brevemente os propósitos do estudo. Foram excluídas as pacientes portadoras de amputações ou com dificuldade de deambular devido às possíveis influências na estimativa da composição corporal.

O DXA foi utilizado como padrão-ouro para avaliação da composição corporal ${ }^{15}$. Com o DXA também se estima o peso corporal pelo cálculo de área e densidade específica dos tecidos. Foram adotados procedimentos padrões para o posicionamento em decúbito dorsal das voluntárias durante a fase de realização do exame, sem objetos de metal ou sapatos. O exame foi realizado pela varredura total do corpo da paciente. Por meio desse rastreamento foram obtidas as imagens e analisadas pelo software específico do equipamento. $\mathrm{O}$ aparelho foi diariamente calibrado, utilizando-se um objeto padrão próprio para massa óssea e outro para as partes moles ${ }^{11}$.

O peso e a estatura eram medidos pela manhã, após o esvaziamento vesical (balança de plataforma Filizola Eletrônica ID 1500; São Paulo, SP: Brasil), com precisão de $0,1 \mathrm{~kg}$ e $0,1 \mathrm{~cm}$, respectivamente. As dobras cutâneas foram medidas utilizando-se um adipômetro científico da marca 
Lange (Beta Technology, Santa Cruz, CA, USA), com pressão constante de $10 \mathrm{~g} / \mathrm{mm}^{2}$ na superfície de contato e precisão de $1 \mathrm{~mm}$, com escala de 0 a $65 \mathrm{~mm}$. O valor considerado foi a média de três medidas, desde que não houvesse variação maior que $3 \mathrm{~mm}$ entre elas. Foram medidas as dobras cutâneas do tríceps, da suprailíaca, e da coxa, de acordo com técnicas padronizadas por Pollock et al. ${ }^{16}$ e utilizadas para obtenção da densidade corporal (DC, g/mL, Equação 1), e posteriormente para o cálculo do percentual de gordura corporal (\%GC, Equação 2) pela fórmula de Siri' ${ }^{18}$ (Fett et al. $\left.{ }^{17}\right)$ :

Equação 1. $D C=1,0994921-(0,0009929 x$ $X)+\left(0,0000023 x X^{2}\right)-(0,0001392 x Y)$

Em que DC, densidade corporal $(\mathrm{g} / \mathrm{mL}) ; X$, soma das dobras cutâneas do tríceps, suprailíaca e coxa em mm; $Y$, idade em anos.

Equação 2. \%GCA=[(4,95/DC) - 4,5]x100

Em que \%GCA é o percentual de gordura corporal pela antropometria.

Foi usado o teste de Kolmogorov e Smirnov para avaliar a normalidade de amostra. Para a análise de correlação, foi utilizada a correlação linear de Pearson e para comparação das médias, foi utilizado teste $t$ de Student para amostras não pareadas. Foram considerados significantes os valores de $p \leq 0,05$ e intervalo de confiança de $95 \%{ }^{19}$. O cálculo para estipular os limites superiores e inferiores da concordância entre os métodos foi realizado pelo procedimento de Bland e Altman ${ }^{20}$, utilizando as equações 3 e 4 .

Equação 3. $L S u p=M D i f+D P_{D i f}$

Em que LSup, limite superior; MDif, média da diferença entre os valores da antropometria menos os do DXA; $\mathrm{DP}_{\mathrm{Dif}}$, desvio-padrão da diferença entre antropometria e DXA.

Equação 4. $\operatorname{LInf}=M D i f-2 \times D P_{D i f}$

Em que LInf, limite inferior.

Entretanto, foi estabelecida uma faixa de valores mais estreita considerando mais ou menos o coeficiente de variação ${ }^{19}$ do DXA (Equação 5). Para isso foram sorteadas seis voluntárias e esta- belecido o coeficiente de variação do DXA para cada variável. Para serem considerados biologicamente correspondentes, os valores da diferença entre os métodos para a variável em questão deveriam estar dentro dessa faixa (Tabela 1).

$$
\text { Equação 5. } \mathrm{CV}=\left(\frac{\mathrm{DP}}{\mathrm{M}}\right) \times 100
$$

Em que CV é o coeficiente de variação; Desvio-Padrão (DP), de cinco medidas consecutivas; média (M) das cinco medidas consecutivas.

O projeto foi aprovado pelo Comitê de Ética em Pesquisa do HCFMRP-USP (HCRP $n^{\circ}$ 9472/2005) e as voluntárias assinaram termo de consentimento livre e esclarecido.

\section{RESULTADOS}

Perfil das voluntarias: o IMC variou de 19 a $43(\mathrm{M}=30, \mathrm{DP}=5) \mathrm{kg} / \mathrm{m}^{2}$ - da normalidade, próximo do deficit de peso a obesidade mórbida; a faixa de idade era de 60 a $70(M=65, D P=3)$ anos de idade; peso de 40 a $108(M=71, D P=14) \mathrm{kg}$; altura de 1,39 a $1,72(\mathrm{M}=1,5, \mathrm{DP}=0,06) \mathrm{m}$. As médias obtidas pelos dois métodos para as diferentes variáveis comparadas não apresentavam diferença estatística (Tabela 2).

Pela análise de Bland \& Altman ${ }^{20}$ foi estimado que a antropometria em média apresentava diferença em relação ao DXA de 2,3kg para MCM de $-0,93 \mathrm{~kg}$ para a MCG e de $-2,7 \%$ para $\%$ GC. Esses valores, porém, estavam dentro do coeficiente de variação do DXA, que era o limite aceitável de comparação entre os métodos (Figuras 1 a 3). Considerando os valores individualmente, o percentual de voluntárias que estava

Tabela 1. Coeficiente de variação das medidas obtidas pelo DXA. Ribeirão Preto (SP), 2004.

\begin{tabular}{lccc}
\hline Medidas & ${ }^{*} \mathrm{CV}$ - mínimo - máximo & $\mathrm{M}$ & $\mathrm{DP}$ \\
\hline Peso $(\mathrm{kg})$ & $0,3-0,5$ & 0,3 & 0,1 \\
Massa corporal magra $(\mathrm{kg})$ & $0,6-6,5$ & 2,9 & 1,9 \\
Massa corporal gorda $(\mathrm{kg})$ & $1-9,6$ & 4,9 & 3,1 \\
Porcentagem de gordura & $0,9-9,3$ & 4,7 & 3,1 \\
\hline
\end{tabular}

${ }^{*}$ Coeficiente de variação (CV); n=6; M: média; DP: desvio-padrão. 
dentro dos limites considerados aceitáveis para cada uma das variáveis da composição corporal era de $45 \%$ para MCM, $72,5 \%$ para a MCG e $53 \%$ para o \%GC.

Todos os componentes da composição corporal estimados pela antropometria e pelo DXA foram significativamente correlacionados (Figuras 1,2,3; peso (kg), $r=0,99, p<0,0001$ ).

\section{DIS CUSS Ã O}

A equação de Pollock et al..$^{16}$ para DC foi utilizada para o cálculo da composição corporal a partir das medidas das dobras cutâneas, considerando ter sido derivada de um amplo espectro populacional e também pela falta de uma equação específica para a população do presente estudo. Outro aspecto importante para essa escolha

Tabela 2. Comparação entre as medidas da antropometria e DXA (Média e Desvio-Padrão). Ribeirão Preto (SP), 2004.

\begin{tabular}{lccccc}
\hline \multirow{2}{*}{ Variáveis } & \multicolumn{2}{c}{ Antropometria } & \multicolumn{2}{c}{ DXA } & \multirow{2}{*}{$P$} \\
\cline { 2 - 5 } & Média & Desvio-Padrão & Média & Desvio-Padrão & 0,370 \\
\hline Peso $(\mathrm{kg})^{\star}$ & 71 & 14 & 70 & 14 & 0,065 \\
Massa magra $(\mathrm{kg})^{* *}$ & 44 & 6 & 42 & 7 & 0,621 \\
Massa gorda $(\mathrm{kg})^{* *}$ & 27 & 11 & 28 & 5 & 0,051 \\
\% Gordura total ${ }^{* *}$ & 37 & 9 & 39 & 5 & \\
\hline
\end{tabular}

*Mann-Whitney; ${ }^{* *}$ Teste $t$ de Student.

DXA: Raios-X de dupla varredura; $\mathrm{n}=51$.
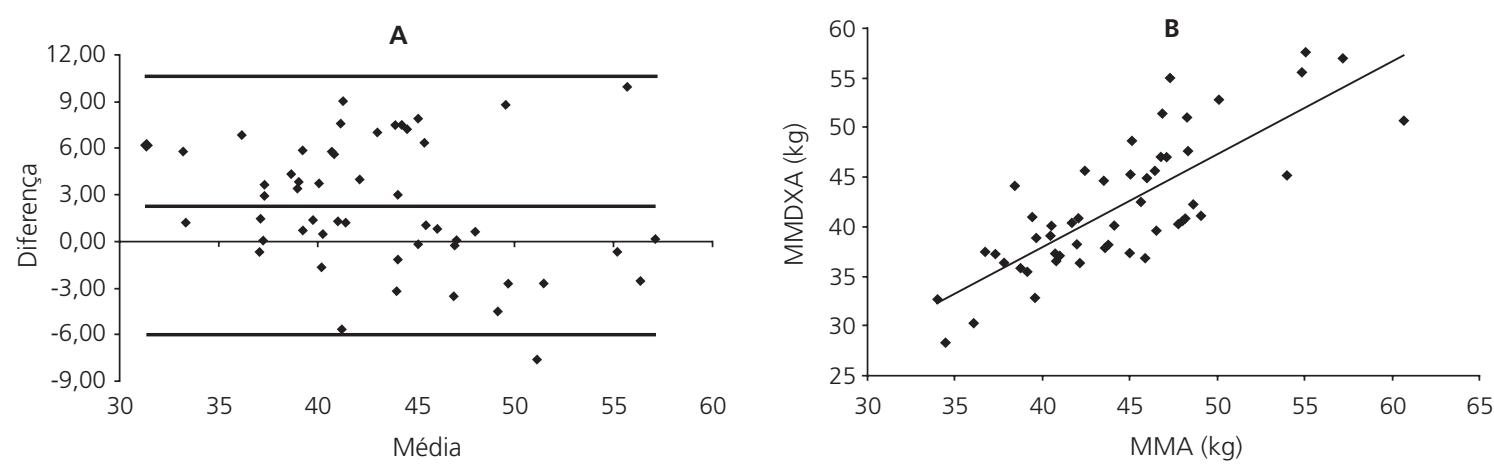

Figura 1. A) A concordância entre a massa magra estimada pela antropometria e pelo DXA demonstra viés de $+2,31 \mathrm{~kg}$ para a antropometria. B) Correlação linear de Pearson entre massa magra estimada pelos métodos citados acima ( $n=51 ; r=0,79$; p<0,0001). Ribeirão Preto (SP), 2004.
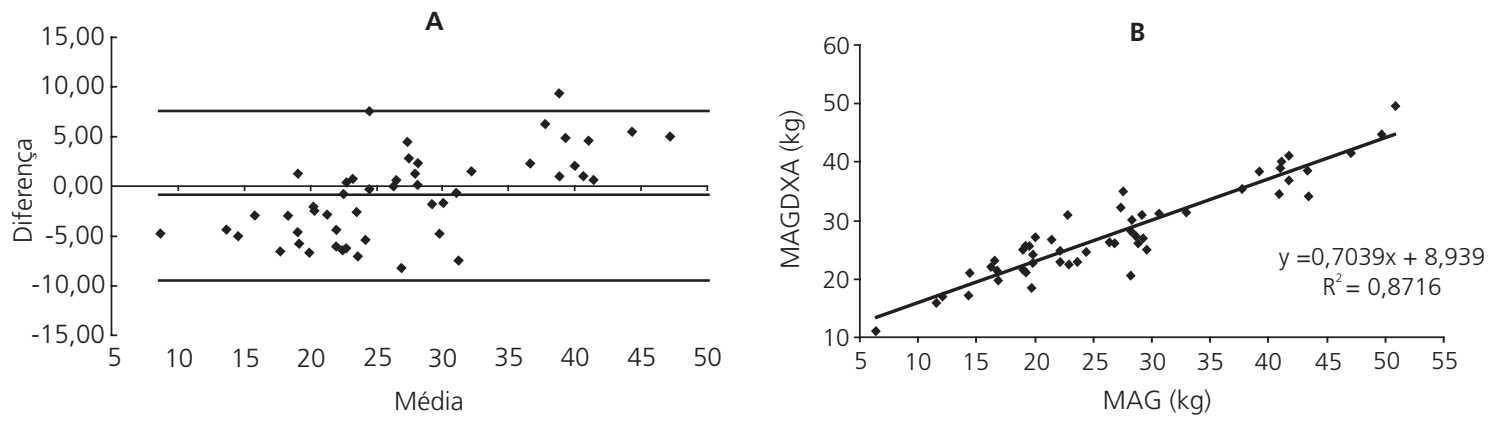

Figura 2. A) A concordância entre a massa magra estimada pela antropometria e pelo DXA demonstra viés de $-0,93 \mathrm{~kg}$ para a antropometria. B) Correlação linear de Pearson entre a massa gorda estimada pela antropometria e $D X A(n=51 ; r=0,93$; p<0,0001). Ribeirão Preto (SP), 2004. 
$700 \mid$ W.C.R. FETT et al.
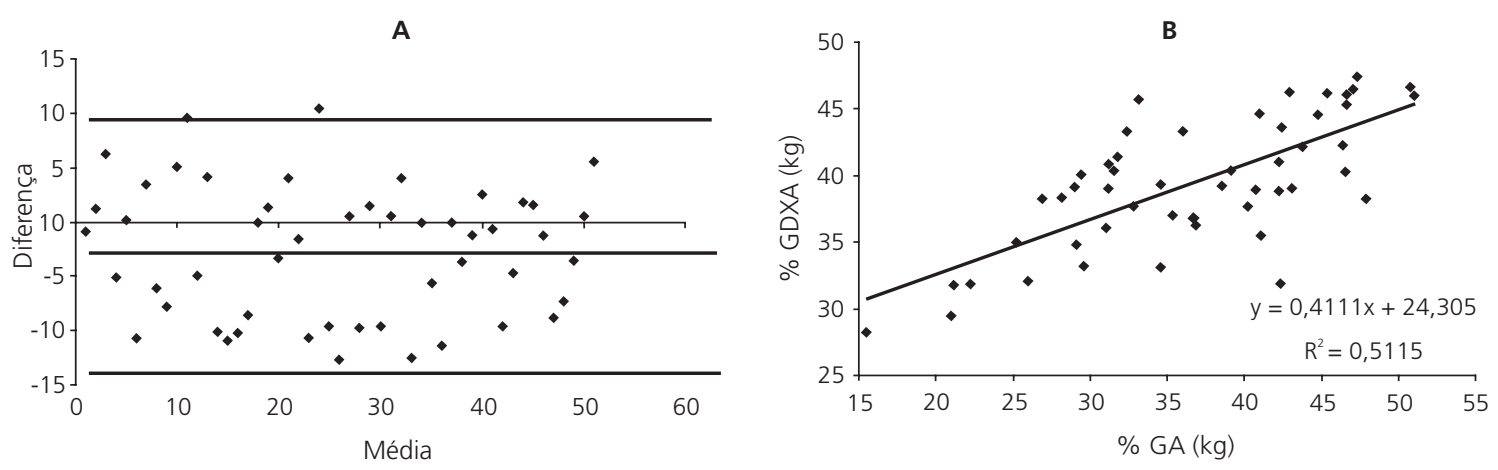

Figura 3. A) A concordância entre a massa magra estimada pela antropometria e pelo DXA demonstra viés de $-2,7 \%$ para a antropometria. B) Correlação linear de Pearson entre o percentual de gordura ( $n=51 ; r=0,72 ; p<0,0001)$. Ribeirão Preto (SP), 2004.

foi o uso da idade na equação, que é um importante fator de correção em modelo de regressão múltipla ${ }^{21}$. Ainda, havia o interesse em observar se essa equação era aplicável nessa população, tendo um padrão-ouro como referência. Por sua vez, o DXA foi escolhido como referência pela facilidade de aplicação e pela fidedignidade e reprodutibilidade do teste ${ }^{11,12,13}$.

Rodrigues Barbosa et al..$^{8}$ observaram que ao utilizar diferentes equações para estimativa do \%GC em mulheres idosas, a antropometria e a bioimpedância subestimavam essa variável comparada ao DXA, com exceção de uma das equações para bioimpedância. Já estudo realizado por De Lorenzo et al..$^{22}$ com adolescentes moderadamente ativos, utilizando-se diferentes equações para antropometria e a bioimpedância comparadas ao DXA, não foi observada diferença para estimativa do \%GC, mas a maioria das equações subestimavam a MCM, ao contrário do observado no presente estudo. Esses autores ${ }^{8,22}$ concluíram que os resultados dos métodos podem variar de acordo com a equação utilizada. Entretanto, no presente estudo, essas diferenças não foram significativas, estavam dentro do limite aceitável para o CV e eram altamente correlacionadas. Além disso, as mudanças na estrutura muscular e no tecido adiposo dessas idosas devem ter contribuído para que a MCM fosse superestimada.

A antropometria apresentou correlação significativa com a ressonância magnética em ido$\operatorname{sos}(r=0,90)^{21}$, teve boa concordância com o DXA em 731 adultos entre 50 e 79 anos de idade ${ }^{23}$ e não apresentou diferença entre as médias comparadas pela bioimpedância em idosas ${ }^{8}$ e em obesas $^{17}$. Por outro lado, estudos demonstraram que a antropometria apresentava discrepância em relação a outros métodos, como a baixa concordância com a bioimpedância e em relação ao DXA subestimava o \% $\mathrm{GC}^{8}$ e a $\mathrm{MCM}^{22}$. Outros métodos mais elaborados também apresentam diferenças entre suas estimativas. Minderico et al. ${ }^{24}$ verificaram em 93 mulheres saudáveis que havia diferença significativa entre a pletismografia e o DXA, mas os métodos eram concordantes entre si. Porém, a MCG e o \%GC eram superestimados pela bioimpedância em crianças $^{25}$ e em obesas de meia idade ${ }^{26}$ e pela pletismografia em mulheres saudáveis $^{24}$ quando comparados ao DXA.

No presente estudo, embora as médias dos valores das variáveis para composição corporal fossem iguais entre si e tenham ficado dentro dos limites aceitáveis para a concordância, aproximadamente metade da amostra ficava fora desses limites para MCM e para o \%GC, sugerindo cautela em sua equivalência. Por outro lado, considerando a antropometria o recurso mais simples de avaliação da composição corporal, a população do presente estudo, mais complicada que as de vários estudos citados e as controversas existentes na literatura, os resultados obtidos aqui podem ser considerados satisfatórios.

Nos indivíduos idosos, as medidas dos componentes corporais podem apresentar algu- 
mas limitações devido à redistribuição da gordura subcutânea, à atrofia dos adipócitos e à diminuição da elasticidade da pele, podendo ocasionar maior compressão entre a gordura e a massa muscular, o que afeta de forma significativa a confiabilidade das estimativas da gordura corporal por meio da antropometria ${ }^{8}$, pois pode fornecer uma medida menor da dobra cutânea. Mesmo assim, no presente estudo, os resultados obtidos pela antropometria não diferiram muito dos do DXA. Portanto, como discutido acima, os resultados para composição corporal obtidos por diferentes métodos são conflitantes na literatura ${ }^{8,12,21-24} \mathrm{e} \mathrm{a}$ equivalência entre os métodos e as populações é limitada. Diferentes populações, protocolos e metodologias podem responder, ao menos em parte, por essas variações.

Portanto, mesmo com alta correlação, a concordância e a comparação das médias podem apresentar diferenças entre os métodos de avaliação da composição corporal ${ }^{20}$. Assim, os resultados do presente estudo, que relaciona três métodos estatísticos, foram consistentes. É necessário, ainda, considerar que foi utilizado o coeficiente de variação do DXA como limite aceitável para comparação com a antropometria. Nesse ponto, o DXA, um método mais acurado que a antropometria e aqui considerado padrão ouro para estimativa da composição corporal, apresentou variações biologicamente importantes. Assim, se o DXA - método muito mais complexo e de alta tecnologia - apresentou essas variações e a antropometria um procedimento duplamente indireto e muito mais barato produziu resultados aproximados aos do DXA, o resultado aqui obtido pode ser considerado relevante.

\section{O N CLUS Ã O}

Embora seja limitada a intercambialidade entre os métodos de estimativa da composição corporal e seja necessário interpretá-los com precaução, métodos mais simples são ferramentas úteis para estudos em grande escala. Considerando a alta correlação observada no presente estudo, a igualdade entre as médias e a diferença dentro do limite estipulado como aceitável, é sugerido que, para o nível populacional, a antropometria possa ser consistente para a avaliação da composição corporal comparada ao DXA nesse grupo de idosas diabéticas. Pretende-se com futuros estudos com métodos considerados padrão- ouro e amostras maiores contribuir para o esclarecimento das questões observadas no presente estudo e ainda não respondidas.

\section{A GRADECIMENTOS}

Ao Prof Dr. Francisco J.A. de Paula do Departamento de Radiologia responsável técnico do DXA, pela orientação técnica; ao Dr. Milton C. Foss pela disponibilização do local para seleção das voluntárias; ao Dr. Antonio D. Campos do Departamento de Serviço Social pela sugestão do método para análise estatística.

\section{OLABORADORES}

W.C.R. FETT escreveu o projeto para aprovação do comitê de ética em pesquisa; W.C.R. FETT e C.A. FETT coletaram todos os dados do estudo e escreveram a versão final do manuscrito; J.S. MARCHINI revisou o projeto e a versão final do manuscrito. J.C. MORIGUTI discutiu sobre a comparação dos métodos e importância para esta população; E. FERRIOLLI revisou e orientou o trabalho do início a conclusão.

\section{REFERÊ N CIAS}

1. Reaven GM. Banting lecture: role of insulin resistance in human disease. Diabetes. 1988; 37(12):1595-607. doi:10.1016/0899-9007(97)90 878-9.

2. Hays NP, Galassetti PR, Coker RH. Prevention and treatment of type 2 diabetes: current role of lifestyle, natural product, and pharmacological interventions. Pharmacol Ther. 2008; 118(2):181-91. doi:10.1016/j.pharmthera.2008.02.003.

3. Horton E, Cefalu WT, Haines ST, Siminerio LM. Multidisciplinary interventions: mapping new horizons in diabetes care. Diabetes Educ. 2008; 34(Supp|4):78S-89S. doi: 10.1177/01457217083 21148.

4. Sartorelli DS, Franco LJ. ências do diabetes mellitus no Brasil: o papel da transição nutricional. Cad Saúde Pública. 2003; 19(1):29-36. doi:10.1590/S0 102-311X2003000700004 
702 | W.C.R. FETT et al.

5. Wild S, Roglic G, Green A, Sicree S, King H. Global prevalence of diabetes: estimates for the year 2000 and projections for 2030. Diabetes Care. 2004; 27(5):1047-53. doi:10.2337/diacare.27.5.1047.

6. Malerbi DA, Franco LJ. Multicenter study of the prevalence of diabetes mellitus and impaired glucose tolerance in the urban Brazilian population aged 30-69 yr. The Brazilian Cooperative Group on the Study of Diabetes Prevalence. Diabetes Care. 1992; 15(11):1509-16. doi:10.2337/diacare.15.11. 1509.

7. Torquato MT, Montenegro Júnior RM, Viana LA, de Souza RA, Lanna CM, Lucas JC, et al. Prevalence of diabetes mellitus and impaired glucose tolerance in the urban population aged 30-69 years in Ribeirão Preto (São Paulo), Brazil. São Paulo Med J. 2003; 121(6):224-30. doi:10.1590/\$1516-3180 2003000600002.

8. Rodrigues Barbosa A, Santarém JM, Jacob Filho W, Meirelles ES, Nunes Marucci MF. Comparação da gordura corporal de mulheres idosas segundo antropometria, bioimpedância e DEXA. Arch Latinoam Nutr. 2001; 51(1):49-56.

9. King $\mathrm{H}$, Rewers $\mathrm{M}$. Global estimates for prevalence of diabetes mellitus and impaired glucose tolerance in adults. Diabetes Care. 1993; 16(1):157-77. doi: 10.2337/diacare.16.1.157.

10. Villareal DT, Banks M, Siener C, Sinacore DR, Klein S. Physical frailty and body composition in obese elderly men and women. Obes Res. 2004; 12(6): 913-20. doi: 10.1038/oby.2004.111.

11. Genton L, Hans D, Kyle UG, Pichard C. Dual-energy X-ray absorptiometry and body composition: differences between devices and comparison with reference methods. Nutrition. 2002; 18(1):66-70. doi:10.1016/S0899-9007(01)00700-6.

12. Svendsen OL. Should measurement of body composition influence therapy for obesity? Acta Diabetol. 2003; 40(1):S250-3. doi:10.1007/ s00592-003-0078-y.

13. Visser $M$, Fuerst $T$, Lang $T$, Salamone $L$, Harris TB. Validity of fan-beam dual-energy $X$-ray absorptiometry for measuring fat-free mass and leg muscle mass. J Appl Physiol. 1999; 87(4): 1513-20.

14. Ball SD, Altena TS, Swan PD. Comparison of anthropometry to DXA: a new prediction equation for men. Eur J Clin Nutr. 2004; 58(11):1525-31. doi:10.1038/sj.ejcn.1602003.

15. Salamone LM, Fuerst $T$, Visser M, Kern M, Lang $T$, Dockrell $\mathrm{M}$, et al. Measurement of fat mass using DXA: a validation study in elderly adults. J Appl Physiol. 2000; 89(1):345-52.

16. Pollock ML, Schmidt DH, Jackson AS. Measurement of cardio respiratory fitness and body composition in the clinical setting. Comp Ther. 1980; 6(9):12-27.
17. Fett CA, Fett WCR, Oyama SR, Marchini JS. Composição corporal e somatótipo de mulheres com sobrepeso e obesas pré e pós-treinamento em circuito ou caminhada. Rev Bras Med Esporte 2006;12(1):45-50. doi:10.1590/\$1517-86922006 000100009.

18. Siri SE. Body composition from fluid spaces and density: analysis of methods. In: Brozek J, Henschel A, editors. Techniques for measuring body composition. Washington, DC: National Academy of Sciences, National Research Council; 1961. p. 223-244.

19. Dawson B, Trapp RG. Basic and clinical biostatistics. $2^{\text {nd }}$ ed. Connecticut: McGraw-Hill; 1994. p.82-97, 99-122, 162-183.

20. Bland JM, Altman DG. Statistical methods for assessing agreement between two methods of clinical measurement. Lancet. 1986; 327(8476): 307-10. doi:10.1016/S0140-6736(86)90837-8.

21. Heymsfield SD, Nunez C, Testolin C, Gallagher D. Anthropometry and methods of body composition measurement for research and field application in the elderly. Eur J Clin Nutr. 2000; 54(3):S26-32.

22. De Lorenzo A, Bertini I, Candeloro N, lacopino L, Andreoli A, Van Loan MD. Comparison of different techniques to measure body composition in moderately active adolescents. Br J Sports Med. 1998; 32(3):215-9. doi:10.1136/bjsm.32.3.215.

23. Shaw KA, Srikanth VK, Fryer JL, Blizzard L, Dwyer T, Venn AJ. Dual energy X-ray absorptiometry body composition and aging in a population-based older cohort. Int J Obes. 2007; 31(2):279-84. doi:10.1038/sj.ijo.0803417.

24. Minderico CS, Silva AM, Teixeira PJ, Sardinha LB, Hull HR, Fields DA. Validity of air-displacement plethysmography in the assessment of body composition changes in a 16-month weight loss program. Nutr Metab. 2006; 3:32. doi:10.1186/ 1743-7075-3-32.

25. Hosking J, Metcalf BS, Jeffery AN, Voss LD, Wilkin TJ. Validation of foot-to-foot bioelectrical impedance analysis with dual-energy X-ray absorptiometry in the assessment of body composition in young children: the Early Bird cohort. Br J Nutr. 2006; 96(6):1163-8. doi:10.1017/ BJN20061960.

26. Neovius M, Hemmingsson E, Freyschuss B, Udden J. Bioelectrical impedance underestimates total and truncal fatness in abdominally obese women. Obesity. 2006; 14(10):1731-8. doi:10.1038/oby. 2006.199.

Recebido em: 23/10/2008 Versão final reapresentada em: 26/5/2010 Aprovado em: 5/7/2010 